\title{
Efficacy and safety of thalidomide in patients with hepatocellular carcinoma
}

\author{
Hsueh-Erh Chiou, Tsang-En Wang, Ying-Yue Wang, Hui-Wen Liu
}

\begin{abstract}
Hsueh-Erh Chiou, Ying-Yue Wang, Hui-Wen Liu, Pharmacy Department, Mackay Memorial Hospital, Taipei, Taiwan

Tsang-En Wang, Gastroenterology Section, Department of Internal Medicine, Mackay Memorial Hospital, Taipei, Taiwan Correspondence to: Hui-Wen Liu, MS, Pharmacy Department, Mackay Memorial Hospital, 92, Sec 2, Chung-Shan North Road, Taipei 10449, Taiwan. rose211@ms1.mmh.org.tw

Telephone: +886-2-25433535-3284 Fax: +886-2-25433535-3348

Received: 2005-04-22 Accepted: 2005-06-02
\end{abstract}

\begin{abstract}
AIM: To evaluate which patients with hepatocellular carcinoma (HCC) are most likely to respond to thalidomide treatment.

METHODS: From July 2002 to July 2004, patients with HCC who received thalidomide treatment, were enrolled. We extracted relevant data from the patients' medical records, including history and type of hepatitis, comorbidity, serum $\alpha$-fetoprotein ( $\alpha$-FP) level, volumetric changes in tumor, length of survival, and the dose, duration, side effects of thalidomide treatment. The tumor response was evaluated. On the basis of these data, the patients were divided into two groups: those with either partial response or stable disease (PR + SD group) and those with progressive disease (PD group).
\end{abstract}

RESULTS: Two of 42 (5\%) patients had a partial tumor response after treatment with thalidomide, $200 \mathrm{mg} / \mathrm{d}$, and $9(21 \%)$ had stable disease. Patients in the PR + SD group all had cirrhosis. Comparing patients with and without cirrhosis, the former were more likely to respond to thalidomide therapy (PR + SD: $100 \%$ vs PD: $64.5 \%, P=0.041<0.05)$. Thalidomide was significantly more likely to be effective in tumors smaller than $5 \mathrm{~cm}$ (PR + SD: $63.6 \%$ vs PD: $25.8 \%, P=0.034<0.05$ ). Compared with patients with progressive disease (PD), patients in the PR + SD group had a higher total dose of thalidomide $(13669.4 \pm 8446.0 \mathrm{mg}$ vs $22022.7 \pm$ $11461.4 \mathrm{mg}, P=0.023<0.05)$ and a longer survival $(181.0 \pm 107.1 \mathrm{~d}$ vs $304.4 \pm 167.1 \mathrm{~d}, P=0.047<0.05)$. Patients with comorbid disease had a significantly greater incidence of adverse reactions than those without $(93.8 \%$ vs $60.0 \%, P=0.021<0.05)$. The average number of adverse reactions in each person with a comorbid condition was twice as high as in those without other diseases $(2.2 \pm 1.3$ vs $1.1 \pm 1.2 ; P=0.022<0.05)$.

CONCLUSION: Thalidomide therapy is most likely to be effective in patients with early stage small HCC, espe- cially in those with other underlying diseases. A low dose $(200 \mathrm{mg} / \mathrm{d})$ of thalidomide is recommended to continue the treatment long enough to make it more effective.

(c) 2006 The WJG Press. All rights reserved.

Key words: Thalidomide; Hepatocellular carcinoma; Antiangiogenic agents; Adverse events

Chiou HE, Wang TE, Wang YY, Liu HW. Efficacy and safety of thalidomide in patients with hepatocellular carcinoma. World J Gastroenterol 2006; 12(43): 6955-6960

http://www.wjgnet.com/1007-9327/12/6955.asp

\section{INTRODUCTION}

Hepatocellular carcinoma (HCC) is the fifth most prevalent cancer in the world. About one million people die of it each year ${ }^{[1,2]}$. Particularly in Taiwan, it poses a tremendous threat to citizens' health and lives. HCC has been the leading cause of cancer death in Taiwan for the past two years, leading to about 6000 to 7000 deaths each year.

Potentially curative therapies for HCC include surgical resection, liver transplantation, and percutaneous ablation. However, HCC is often diagnosed at an advanced stage when curative therapy is likely to be of little value. Thus, most patients are candidates for palliative treatment, which includes transarterial embolization (with or without chemotherapy), hormonal therapy, interferon therapy, etc. Transarterial embolization has been shown to improve survival $^{[1]}$. The response rate to systemic chemotherapy is generally less than $30 \%{ }^{[1]}$, and patients often tolerate it poorly. A further difficulty is that about $80 \%$ of patients with HCC also have cirrhosis, which severely limits the utility of both surgery and chemotherapy. Therefore, we urgently need new therapeutic approaches for HCC.

In 1971, Dr. Judah Folkman suggested using antiangiogenic agents to inhibit tumors, a proposal which has made a new form of anticancer treatment possible. Cancer cells whose blood supply is disrupted are starved of nutrients and oxygen, leading to atrophy and death. Endothelial cells play a key role in angiogenesis, forming the external theca of new blood vessels and possessing a strong ability to reproduce and migrate. Proteins known to activate endothelial cells include vascular endothelial growth factor (VEGF) and basic fibroblast growth factor 
(BFGF), which are regarded as important contributors to the growth of new blood vessels. HCC is a hypervascular tumor, with high concentrations of VEGF and BFGF in tumor cells and blood ${ }^{[3,4]}$. It is therefore reasonable to infer that anti-angiogenesis may inhibit the growth of HCC.

A number of anti-angiogenic agents have been developed, some of which are in phase II or III clinical trials. Thalidomide is such a drug. It was widely used in the 1950 s as a sedative and anti-emetic agent but was found to be teratogenic. Its use has been therefore prohibited in Europe and America. However, in recent years, thalidomide has been found to have many pharmacological properties, including inhibition of angiogenesis and inflammation and regulation of immunity. Thalidomide apparently blocks factors such as VEGF and BFGF of cancer cells ${ }^{[5,6]}$, thereby inhibiting angiogenesis. In some cancers strongly dependent on angiogenesis, such as Kaposi's sarcoma and metastatic prostate cancer, thalidomide has been used with some success ${ }^{[7,8]}$. HCC, also strongly dependent on angiogenesis, is treated with thalidomide in Taiwan.

As with any newly proposed therapy, it is important to identify those patients most likely to benefit from the treatment while minimizing the risk of adverse effects. This understanding may also facilitate conservation of medical resources when the drug is unlikely to help. We designed this study to evaluate the efficacy and adverse effects of thalidomide in HCC by retrospectively reviewing the results in a series of patients in order to identify those most likely to benefit from the treatment while avoiding serious adverse effects.

\section{MATERIALS AND METHODS}

\section{Patients}

From July 2002 to July 2004, 159 patients with HCC were treated with thalidomide. All the patients were informed of the necessity of contraception and the benefits and risks of thalidomide treatment. They gave their informed consent to receive it by the Medicine Advisory Committee of Mackay Memorial Medical Center and the Department of Public Health in Taiwan. HCC was diagnosed by histologic examination, imaging findings (abdominal sonography and computed tomography), or a serum $\alpha$-fetoprotein ( $\alpha$-FP) level greater than $400 \mathrm{ng} / \mathrm{mL}$. The disease was staged by the pathologic tumor-node-metastasis (pTNM) system, the Okuda system for HCC, and the Cancer of the Liver Italian Program (CLIP) scoring system ${ }^{[9-11]}$. We extracted relevant data from the patients' medical records, including age, gender, history and type of hepatitis, comorbidity, duration of HCC, serum $\alpha$-FP level, volumetric changes in tumor, length of survival, and the dose, duration, side effects of thalidomide treatment. Patients were excluded from analysis if they took thalidomide for less than one month, had other therapy after thalidomide, or if the data were inadequate in the record to define the stage or treatment response.

\section{Evaluation of tumor response}

The tumor response was evaluated according to the imaging findings one month before and after admini- stration of thalidomide according to the new version of the Response Evaluation Criteria in Solid Tumor (RECIST) Guidelines $^{[12]}$, as well as $\alpha$-FP levels before and after thalidomide treatment. On the basis of these data, the patients were divided into two groups: those with either partial response or stable disease $(\mathrm{PR}+\mathrm{SD}$ group) and those with progressive disease (PD group).

\section{Thalidomide therapy}

Thalidomide (50 mg tablet marketed as Thado ${ }^{\circledR}$, Taiwan Tung Yang Biopharm Co. Ltd., Taipei) in a dose of 50 to $200 \mathrm{mg} / \mathrm{d}$, was give twice daily in the morning and evening. The dose was adjusted according to the clinical response and side effects. Thalidomide-related toxicity was classified by the Common Terminology Criteria for Adverse Events v 3.0 (CTCAE) established by the National Cancer Institute $(\mathrm{NCI})^{[13]}$.

\section{Statistical analysis}

The clinical characteristics, dose of thalidomide, treatment duration, and length of survival of the two groups were compared by using the Mann Whitney $U$ test and Fisher's exact test. The relation between comorbidity and adverse reactions was examined by Fisher's exact test. $P<0.05$ was considered statistically significant. The overall survival was calculated by the Kaplan-Meier method from the time of the first dose of thalidomide to the patient's death or the last follow-up. The statistical software package used was SPSS (version 10).

\section{RESULTS}

\section{Patients}

Of the 159 patients with HCC treated with thalidomide, 57 took thalidomide for less than one month, and 35 were given other treatment in addition to thalidomide. The data for 25 patients were incomplete, leaving records for 42 patients that were available for analysis. The clinical characteristics are shown in Table 1. Thirty-one (74\%) patients were older than 60 years. Cirrhosis was present in 31 , and $32(76 \%)$ had comorbid conditions. The prevalence of hepatitis B (15/42) and C $(11 / 42)$ was similar. Twentyone $(50 \%)$ of the patients had TNM stage III A, and the majority had their liver function classified as Child-Pugh A or Okuda II . The most frequent CLIP score was 3 (15/42).

\section{Tumor Response}

Of the 42 patients, 2 had a partial tumor response to thalidomide at a dose of $200 \mathrm{mg} /$ day and a decrease in $\alpha$-FP level. They both had TNM stage III A disease, Okuda phase I or II, and a CLIP score of 3 or 4 . Another 9 patients had stable disease, 4 of whom also had a fall in serum $\alpha$-FP level. The remaining 31 patients had disease that continued to progress after thalidomide. These results were comparable to those of other studies ${ }^{[14-19]}$ (Table 2), none of which showed an objective tumor response rate above $10 \%$. The mean time to partial response in our patients was $34 \mathrm{~d}$. The median survival of all 42 patients was $319 \mathrm{~d}$ (range, 144 to $494 \mathrm{~d}$ ), the one-year survival was $14 \%$. The median survival of the PR + SD group was 524 $\mathrm{d}$ (range, 218 to $830 \mathrm{~d}$ ) (Figure 1). 
Table 1 Clinical characteristics of 42 patients with HCC

\begin{tabular}{|c|c|c|c|}
\hline Characteristic & $n$ & yr & $\%$ \\
\hline \multicolumn{4}{|l|}{ Age } \\
\hline Median & \multicolumn{3}{|c|}{67} \\
\hline Range & \multicolumn{3}{|c|}{$32-84$} \\
\hline \multicolumn{4}{|l|}{ Sex } \\
\hline Male & 11 & & 26 \\
\hline Female & 31 & & 74 \\
\hline \multicolumn{4}{|l|}{ Comorbidity } \\
\hline Absent & 10 & & 24 \\
\hline Present & 32 & & 76 \\
\hline \multicolumn{4}{|l|}{ Cirrhosis } \\
\hline Absent & 11 & & 26 \\
\hline Present & 31 & & 74 \\
\hline \multicolumn{4}{|l|}{ Type of hepatitis } \\
\hline Hepatitis B & 15 & & 36 \\
\hline Hepatitis C & 11 & & 26 \\
\hline Hepatitis B + hepatitis C & 1 & & 2 \\
\hline Hepatitis B + hepatitis D & 1 & & 2 \\
\hline No record & 14 & & 33 \\
\hline \multicolumn{4}{|l|}{ TNM stage } \\
\hline I & 2 & & 5 \\
\hline II & 6 & & 14 \\
\hline III A & 21 & & 50 \\
\hline ІІІв & 2 & & 5 \\
\hline IVA & 7 & & 17 \\
\hline IVB & 4 & & 10 \\
\hline \multicolumn{4}{|l|}{ Child-Pugh classification } \\
\hline Grade A & 25 & & 60 \\
\hline Grade B & 14 & & 33 \\
\hline Grade C & 3 & & 7 \\
\hline \multicolumn{4}{|l|}{ Okuda stage } \\
\hline I & 11 & & 26 \\
\hline II & 27 & & 64 \\
\hline III & 4 & & 10 \\
\hline \multicolumn{4}{|l|}{ CLIP score } \\
\hline 0 & 2 & & 5 \\
\hline 1 & 10 & & 24 \\
\hline 2 & 7 & & 17 \\
\hline 3 & 15 & & 36 \\
\hline 4 & 4 & & 10 \\
\hline 5 & 4 & & 10 \\
\hline 6 & 0 & & 0 \\
\hline \multicolumn{4}{|l|}{$\alpha$-fetoprotein } \\
\hline$>400 \mathrm{ng} / \mathrm{mL}$ & 21 & & 50 \\
\hline$\leqslant 400 \mathrm{ng} / \mathrm{mL}$ & 21 & & 50 \\
\hline \multicolumn{4}{|l|}{ Pre thalidomide therapy } \\
\hline $\mathrm{TAE}^{1}$ & 26 & & 62 \\
\hline $\mathrm{PEI}+\mathrm{TAE}^{1}$ & 3 & & 7 \\
\hline Radiation + $\mathrm{TAE}^{1}$ & 2 & & 5 \\
\hline Surgery $+\mathrm{PEI}^{2}$ & 1 & & 2 \\
\hline Chemotherapy & 1 & & 2 \\
\hline No therapy & 9 & & 21 \\
\hline
\end{tabular}

${ }^{1}$ Transarterial embolization; ${ }^{2}$ Percutaneous ethanol injection.

Table 3 shows that all patients in the PR + SD group had cirrhosis, suggesting that cirrhosis might influence the efficacy of thalidomide. Patients in this group were more likely to have a CLIP score of 0 to $2(7 / 11)$ or an $\alpha$-FP level of $<400 \mathrm{ng} / \mathrm{mL}$ (8/11). Response to thalidomide was significantly more likely when the tumor was $<5 \mathrm{~cm}$
Table 2 Response rates of HCC to thalidomide

\begin{tabular}{|c|c|c|c|c|c|c|}
\hline \multirow{2}{*}{ Author } & \multirow{2}{*}{$n$} & \multicolumn{5}{|c|}{ Tumor response, $n$ (\%) } \\
\hline & & $\mathrm{CR}^{1}$ & $\mathbf{P R}^{2}$ & $S D^{3}$ & $\mathrm{PD}^{4}$ & $\mathrm{CR}^{1}+\mathrm{PR}^{2}+\mathrm{SD}^{3}$ \\
\hline Kong et al ${ }^{[14]}$ & 11 & 0 & $1(9)$ & $4(36)$ & $6(55)$ & $5(46)$ \\
\hline Lin $a t$ al ${ }^{[15]}$ & 27 & 0 & $1(4)$ & $1(4)$ & $25(93)$ & $2(7)$ \\
\hline Feun et al ${ }^{[16]}$ & 7 & 0 & 0 & 0 & $7(100)$ & 0 \\
\hline Schwartz et al ${ }^{[17]}$ & 30 & $1(3)$ & $1(3)$ & $9(30)$ & $19(63)$ & $11(37)$ \\
\hline Hsu et al ${ }^{[18]}$ & 63 & $1(2)$ & $3(5)$ & $20(32)$ & $39(62)$ & $24(38)$ \\
\hline Wang et al $l^{[19]}$ & 99 & 0 & $6(6)$ & - & - & - \\
\hline Chiou et al & 42 & 0 & $2(5)$ & $9(21)$ & $31(74)$ & $11(26)$ \\
\hline
\end{tabular}

${ }^{1}$ Complete response; ${ }^{2}$ Partial response; ${ }^{3}$ Stable disease; ${ }^{4}$ Progressive disease.

Table 3 Comparison of patients with partial response or stable disease with patients with progressive disease

\begin{tabular}{|c|c|c|c|}
\hline Characteristic & $\underset{n}{\mathrm{PR}^{1}+\mathrm{SD}^{2}}$ & $\begin{array}{r}P^{3} \\
n\end{array}$ & $P$ \\
\hline Sex & & & 1.0 \\
\hline Male & 8 & 23 & \\
\hline Female & 3 & 8 & \\
\hline Age & & & 0.234 \\
\hline$>60 \mathrm{yr}$ & 10 & 21 & \\
\hline$\leqslant 60 \mathrm{yr}$ & 1 & 10 & \\
\hline Cirrhosis & & & $0.041^{\mathrm{a}}$ \\
\hline Absent & 0 & 11 & \\
\hline Present & 11 & 20 & \\
\hline \multicolumn{4}{|l|}{ Hepatitis } \\
\hline Hepatitis B & 4 & 13 & 1.0 \\
\hline Hepatitis C & 3 & 9 & 1.0 \\
\hline \multicolumn{4}{|l|}{ TNM stage } \\
\hline $\mathrm{I}+\mathrm{II}$ & 3 & 4 & 1.0 \\
\hline III A + III B & 4 & 19 & 0.180 \\
\hline IVA + IVB & 4 & 8 & 1.0 \\
\hline \multicolumn{4}{|c|}{ Child-Pugh classification } \\
\hline Grade A & 5 & 21 & 0.280 \\
\hline Grade B & 6 & 8 & 0.136 \\
\hline Grade C & 0 & 2 & 1.0 \\
\hline \multicolumn{4}{|l|}{ Okuda stage } \\
\hline I & 3 & 8 & 1.0 \\
\hline II & 8 & 19 & 0.717 \\
\hline III & 0 & 4 & 0.558 \\
\hline \multicolumn{4}{|l|}{ CLIP score } \\
\hline $0-2$ & 7 & 13 & 0.298 \\
\hline $3-6$ & 4 & 18 & 0.298 \\
\hline$\alpha$-fetoprotein & & & 0.081 \\
\hline$>400 \mathrm{ng} / \mathrm{mL}$ & 3 & 19 & \\
\hline$<400 \mathrm{ng} / \mathrm{mL}$ & 8 & 12 & \\
\hline Tumor size & & & $0.034^{\mathrm{a}}$ \\
\hline$\leqslant 5 \mathrm{~cm}$ & 7 & 8 & \\
\hline$>5 \mathrm{~cm}$ & 4 & 23 & \\
\hline
\end{tabular}

${ }^{1}$ Partial response; ${ }^{2}$ Stable disease; ${ }^{3}$ Progressive disease; ${ }^{a} P<0.05$ between the two groups.

(PR + SD: $63.6 \%$ vs PD: $25.8 \%, P=0.034<0.05)$. Other clinical characteristics did not differ significantly between the groups.

The duration of thalidomide therapy was longer in the PR + SD group (Table 4). Patients in the PR + SD group had a significantly higher total dose and a significantly longer survival than those in the PD group. 


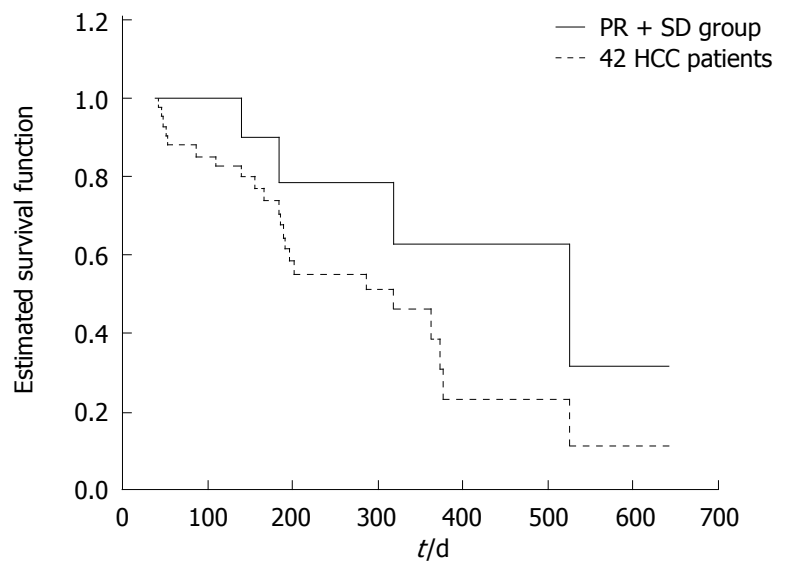

Figure 1 Survival curves in patients with HCC on thalidomide.

\begin{tabular}{|c|c|c|c|}
\hline & $\mathrm{PR}^{1}+\mathrm{SD}^{2}$ & $\mathbf{P D}^{3}$ & $P$ \\
\hline Duration (d) & $167.1 \pm 98.9$ & $107.7 \pm 71.7$ & 0.069 \\
\hline Total dose (mg) & $22022.7 \pm 11461.4$ & $13669.4 \pm 8446.0$ & $0.023^{\mathrm{a}}$ \\
\hline Survival (d) & $304.4 \pm 167.1$ & $181.0 \pm 107.1$ & $0.047^{c}$ \\
\hline
\end{tabular}

${ }^{1}$ Partial response; ${ }^{2}$ Stable disease; ${ }^{3}$ Progressive disease; ${ }^{a} P<0.05,{ }^{c} P<0.05$ vs duration, between the two groups.

Table 5 Thalidomide-related adverse events in patients with $\mathrm{HCC}$

\begin{tabular}{lrrrllr}
\hline & \multicolumn{9}{c}{ Grade of severity $(\boldsymbol{n})$} & & Total \\
Adverse event & $\mathbf{G}^{\mathbf{1}}$ & $\mathbf{G}^{\mathbf{2}}$ & $\mathbf{G}^{\mathbf{3}}$ & $\mathbf{G}^{\mathbf{4}}$ & $\mathbf{G}^{\mathbf{5}}$ & $\boldsymbol{n}(\%)$ \\
\hline Constipation & 2 & 22 & 0 & 0 & 0 & $24(57)$ \\
Skin rash & 6 & 8 & 3 & 0 & 0 & $17(41)$ \\
Low leg edema & 1 & 10 & 0 & 0 & 0 & $11(26)$ \\
Dizziness & 3 & 5 & 1 & 0 & 0 & $9(21)$ \\
Lethargy & 3 & 2 & 2 & 0 & 0 & $7(17)$ \\
Nausea/vomiting & 1 & 5 & 0 & 0 & 0 & $6(14)$ \\
Neurosensory & 3 & 0 & 0 & 0 & 0 & $3(7)$ \\
Dyspnea & 0 & 2 & 0 & 0 & 0 & $2(5)$ \\
Leukopenia & 1 & 0 & 0 & 0 & 0 & $1(2)$ \\
Thrombocytopenic purpura & 0 & 1 & 0 & 0 & 0 & $1(2)$ \\
Insomnia & 0 & 1 & 0 & 0 & 0 & $1(2)$ \\
Alopecia & 1 & 0 & 0 & 0 & 0 & $1(2)$ \\
\hline
\end{tabular}

${ }^{1}$ mild adverse event; ${ }^{2}$ Moderate adverse event; ${ }^{3}$ Severe adverse event; ${ }^{4}$ Lifethreatening or disabling adverse event; ${ }^{5}$ Death related to adverse event.

\section{Thalidomide therapy}

Twenty patients began their treatment with a dose of 200 $\mathrm{mg} / \mathrm{d}$ and the remaining $22100-150 \mathrm{mg} / \mathrm{d}$ initially. Ten patients had their dose escalated to $200 \mathrm{mg}$ according to treatment response, while three patients had their dose reduced because of thalidomide- induced drowsiness, dizziness and constipation, and 2 discontinued it entirely. Most adverse reactions were slight or moderate, only 6 $(14 \%)$ events (skin rash in 3 , dizziness in 1 , and lethargy in 2) were severe. The most common adverse reactions were constipation and skin rash, with an incidence of $57 \%$ and
Table 6 Thalidomide-related adverse events and comorbidity

\begin{tabular}{lcc}
\hline \multirow{2}{*}{ Adverse drug events per patient } & \multicolumn{2}{c}{ Comorbidity $(\boldsymbol{n})$} \\
\cline { 2 - 3 } & Absent & Present \\
\hline 1 & 3 & 10 \\
2 & 1 & 7 \\
3 & 2 & 6 \\
4 & 0 & 7 \\
\hline
\end{tabular}

Table 7 Correlation between thalidomide-related adverse events and comorbidity

\begin{tabular}{|c|c|c|c|}
\hline & \multicolumn{2}{|c|}{ Comorbidity (n) } & \multirow{2}{*}{$P$} \\
\hline & Absent & Present & \\
\hline Without adverse events & 4 & 2 & \multirow{2}{*}{0.021} \\
\hline With adverse drug events ${ }^{a}$ & 6 & 30 & \\
\hline Adverse events per patient ${ }^{c}$ & $1.1 \pm 1.2$ & $2.2 \pm 1.3$ & 0.022 \\
\hline
\end{tabular}

${ }^{\mathrm{a}} P<0.05,{ }^{\mathrm{c}} P<0.05$ vs without adverse events, between groups of with comorbidity and without comorbidity.

$41 \%$, respectively (Table 5).

Tables 6 and 7 show the relationship between thalidomide-induced adverse reactions and comorbidity. Adverse reactions were more likely to occur in patients with an other underlying diseases $(93.8 \%$ vs $60.0 \%, P=$ $0.021<0.05)$. The average number of adverse reactions in each person with a comorbid condition was twice as high as in those without other diseases $(2.2 \pm 1.3$ vs $1.1 \pm 1.2, P$ $=0.022<0.05)$.

\section{DISCUSSION}

In our series, the partial response rate of $\mathrm{HCC}$ to thalidomide at 100 to $200 \mathrm{mg} / \mathrm{d}$ was $5 \%, 21 \%$ of patients who had stabilization of their disease after treatment with thalidomide. These results generally agree with those of other researchers. In our study, two thirds of the patients were older than 60 and had cirrhosis or other comorbid conditions. Most of them had TNM stage III A disease or were in Okuda class II.

The pharmacological mechanisms of thalidomide include anti-angiogenic, immunomodulatory and antiinflammatory effects. The drug inhibits angiogenesis in tumors by blocking the activity of VEGF and BFGF ${ }^{[20]}$. The immunomodulatory effects include induction of TNF- $\alpha$ mRNA degradation, thereby inhibiting the synthesis of TNF- $\alpha^{[21]}$. It also downregulates lymphocyte surface molecules, lowering the CD4:CD8 peripheral lymphocyte ratio. It can inhibit chemotaxis of neutrophils, interleukin- 6 and interleukin-12, and stimulate the synthesis of interleukin- $2^{[22]}$. In addition, it inhibits the activity of $\mathrm{I} \kappa \mathrm{B}$ kinase, thus blocking the activity of nuclear factor- $\kappa \mathrm{B}^{[23]}$.

Because of its many pharmacological actions, thalidomide is now widely used in the treatment of a number of diseases. In 1999, it was approved by the FDA to treat erythema nodosum leprosum. It has been used for aphthous ulcers in HIV-positive patients, graft versus 
host disease after bone marrow grafting, tuberculosis, sarcoidosis, inflammatory bowel disease, rheumatoid arthritis, as well as for some cancers such as multiple myeloma, renal cell carcinoma, brain tumors, prostate cancer, melanoma and Kaposi sarcoma ${ }^{[1,23]}$. The response of multiple myelomas to thalidomide (600 to $800 \mathrm{mg}$ daily) is about $30 \%$, and increases to $60 \%$ when combined with dexamethasone. The therapeutic effect of thalidomide in HCC is thus not as satisfactory as in multiple myeloma. The reported response is $40 \%$ in Kaposi sarcoma and $15 \%-27 \%$ in prostate cancer ${ }^{[23]}$.

In our study, the plasma concentrations of VEGF and BFGF in multiple myeloma remained unchanged irrespective of whether the disease responded to thalidomide. It is likely that there are mechanisms other than the inhibition of angiogenesis that are responsible for the drug's efficacy in multiple myeloma. For example, there are decreased adhesion of multiple myeloma cells to stromal cells, reduced secretion of cytokines, and activation of caspase-8, leading to death of multiple myeloma cells ${ }^{[24]}$.

Because of the different structures and functions of blood vessels in different tissues and tumors at different sites and the genetic instability and biologic heterogeneity of tumor cells, new blood vessels in tumors may be quite diverse. There are therefore considerable complexity and unpredictability of tumor microvasculature ${ }^{[25]}$. This may help explain why thalidomide has different effects on different malignancies. The relatively poor response of HCC compared to multiple myeloma may be related to the differences in angiogenesis.

In our series, only about one fifth of the patients had their disease stabilized after thalidomide therapy, indicating that thalidomide helps only a few patients with advanced HCC, and rarely results in a decrease in tumor size. The median survival in the PR + SD group was 524 d (range, 218-830 d) compared with 319 d (range, 144-494 d) of all 42 patients. The one-year survival was only $14 \%$, suggesting that thalidomide is not very effective in prolonging the life of HCC patients.

Our results did show that patients with cirrhosis tolerated thalidomide well and even had a somewhat better response to treatment. The reason why response in those with tumors smaller than $5 \mathrm{~cm}$ is better may be that thalidomide can prominently inhibit formation of new blood vessels rather than disrupt existing mature vessels. Larger tumors with mature vasculature may therefore be relatively resistant to the action of thalidomide. The maximum therapeutic effect may thus be seen in smaller tumors with newer vessels.

In the PR + SD group $64 \%$ of patients had a CLIP score of 0 to 2 , compared with $42 \%$ in the PD group. Similarly more patients in the PR + SD group $(73 \%)$ had an $\alpha$-FP level of $<400 \mathrm{ng} / \mathrm{mL}$ compared with the PD group $(39 \%)$. The PR + SD group also took a significantly higher total dose of thalidomide $(22022.7 \pm 11461.4 \mathrm{mg}$ vs $13669.4 \pm 8446.0 \mathrm{mg}, P=0.023)$ and had a significantly longer survival $(304.4 \pm 167.1 \mathrm{~d}$ vs $181.0 \pm 107.1 \mathrm{~d} ; P=$ $0.047)$. These results indicate that treatment of earlier stage HCC with thalidomide may leave enough time for the drug to produce a significant therapeutic effect.

A commonly-reported adverse effect in patients treated with thalidomide for multiple myeloma is venous thromboembolism, particularly when combined with doxorubicin or with dexamethasone ${ }^{[27]}$. None of the patients in our series had thromboembolism, perhaps because we used smaller doses than the commonlyused doses for multiple myeloma. The most common adverse reactions in our series were constipation (57\%) and skin rash (41\%). Severe reactions were uncommon (14\%), and only 5 patients required a dose adjustment or discontinuation of treatment. Three patients developed slight peripheral neuropathy, and one of them had a dose adjustment from $200 \mathrm{mg}$ to $100 \mathrm{mg}$. Thalidomide-related peripheral neuropathy may occur in patients on long-term treatment and is irreversible. Therefore, if it occurs, the drug should be stopped or the dose is reduced. Generally, the adverse reactions caused by thalidomide are dose- and duration-related $^{[27]}$. We also found a significant positive correlation between adverse reactions and comorbidity $(P$ $=0.021$ ) of diabetes mellitus, hypertension, ischemic heart disease, peptic ulcer, chronic obstructive lung disease and benign prostatic hyperplasia. In considering therapy for patients with HCC, the higher risk of adverse reactions to thalidomide in the presence of other diseases should be taken into account.

Our study is limited by the fact that it was retrospective. It would be difficult to collect enough patients with HCC in similar stages for comparison in a prospective study. We also were only able to analyze the data from a relatively small number of patients, which might skew the results to some extent. However, thalidomide is unlikely to be effective in a large proportion of patients with HCC. This makes it even more important to identify those who are likely to benefit from thalidomide treatment.

\section{ACKNOWLEDGMENTS}

We thank Pei-Fang Wu and Ming-Min Sun, pharmacists of the Pharmacy Department, Mackay Memorial Hospital, for their assistance and support with data collection and Dr. Mary Jeanne Buttrey, a consulting physician, Department of Internal Medicine, Mackay Memorial Hospital, for critical review and revision of the manuscript.

\section{REFERENCES}

1 Burroughs A, Hochhauser D, Meyer T. Systemic treatment and liver transplantation for hepatocellular carcinoma: two ends of the therapeutic spectrum. Lancet Oncol 2004; 5: 409-418

2 Llovet JM, Burroughs A, Bruix J. Hepatocellular carcinoma. Lancet 2003; 362: 1907-1917

3 Torimura $T$, Sata $M$, Ueno $T$, Kin M, Tsuji R, Suzaku K, Hashimoto O, Sugawara H, Tanikawa K. Increased expression of vascular endothelial growth factor is associated with tumor progression in hepatocellular carcinoma. Hum Pathol 1998; 29: 986-991

4 Yamaguchi R, Yano H, Iemura A, Ogasawara S, Haramaki M, Kojiro M. Expression of vascular endothelial growth factor in human hepatocellular carcinoma. Hepatology 1998; 28: 68-77

5 Kenyon BM, Browne F, D'Amato RJ. Effects of thalidomide and related metabolites in a mouse corneal model of neovascularization. Exp Eye Res 1997; 64: 971-978

6 Peuckmann V, Fisch M, Bruera E. Potential novel uses of thalidomide: focus on palliative care. Drugs 2000; 60: 273-292

7 Little RF, Wyvill KM, Pluda JM, Welles L, Marshall V, Figg WD, Newcomb FM, Tosato G, Feigal E, Steinberg SM, Whitby 
D, Goedert JJ, Yarchoan R. Activity of thalidomide in AIDSrelated Kaposi's sarcoma. J Clin Oncol 2000; 18: 2593-2602

8 Patterson SG, Fishman M, Kish J, Ewbank D, Defelice J, Rago $\mathrm{R}$. Thalidomide plus daily oral dexamethasone for metastatic hormone refractory prostate cancer (HRPC) in patients previously treated with cytotoxic chemothertapy. Proc Am Soc Clin Oncol 2003; 22: 444, abstract 1783

9 Sobin L, Wittekind C. TMN classification of malignant tumours. 5th ed. New York: Wiley-Liss, 1997: 74-77

10 Okuda K. Early recognition of hepatocellular carcinoma. Hepatology 1986; 6: 729-738

11 A new prognostic system for hepatocellular carcinoma: a retrospective study of 435 patients: the Cancer of the Liver Italian Program (CLIP) investigators. Hepatology 1998; 28: 751-755

12 Therasse $\mathbf{P}$, Arbuck SG, Eisenhauer EA, Wanders J, Kaplan RS, Rubinstein L, Verweij J, Van Glabbeke M, van Oosterom AT, Christian MC, Gwyther SG. New guidelines to evaluate the response to treatment in solid tumors. European Organization for Research and Treatment of Cancer, National Cancer Institute of the United States, National Cancer Institute of Canada. J Natl Cancer Inst 2000; 92: 205-216

13 National cancer institute. Cancer Therapy Evaluation Program: Common Terminology Criteria for Adverse Events v3.0 (CTCAE). Published 2003-12-12, cited 2004-8-22. Available from: http://ctep.cancer.gov/reporting/ctc.html

14 Kong HL, Boyer MJ, Lim R, Clarke S, Milward JM, Wong E. Phase II trial of thalidomide in unresectable hepatocellular carcinoma (HCC)-a cancer therapeutics group (CTRG) study. Pro Am Soc Clin Oncol 2001; 20: 133b, abstract 2282

15 Lin AY, Brophy N, Fisher GA, So S, Biggs C, Yock T, Levitt L. Phase II study of thalidomide in patients (pts) with unresectable hepatocellular carcinoma (HCC). Proc Am Soc
Clin Oncol 2002; 21: 97b, abstract 2202

16 Feun LG, Marini A, Molina E, O'Brien C, Schiff E, Jeffers L., Savaraj N. Thalidomide as palliative care for patients with unresectable hepatocellular carcinoma. Proc Am Clin Oncol 2003; 22: 216, abstract 865

17 Schewartz JD, Lehrer D, Mandeli J, Goldenberg A, Sung M, Volm M. Thalidomide in hepatocellular cancer (HCC) with optional interferon-a upon progression. Proc Am Clin Oncol 2003; 22: 301, abstract 1210

18 Hsu C, Chen CN, Chen LT, Wu CY, Yang PM, Lai MY, Lee $\mathrm{PH}$, Cheng AL. Low-dose thalidomide treatment for advanced hepatocellular carcinoma. Oncology 2003; 65: 242-249

19 Wang TE, Kao CR, Lin SC, Chang WH, Chu CH, Lin J, Hsieh RK. Salvage therapy for hepatocellular carcinoma with thalidomide. World J Gastroenterol 2004; 10: 649-653

20 D'Amato RJ, Loughnan MS, Flynn E, Folkman J. Thalidomide is an inhibitor of angiogenesis. Proc Natl Acad Sci USA 1994; 91: 4082-4085

21 Moreira AL, Sampaio EP, Zmuidzinas A, Frindt P, Smith KA, Kaplan G. Thalidomide exerts its inhibitory action on tumor necrosis factor alpha by enhancing mRNA degradation. J Exp Med 1993; 177: 1675-1680

22 Richardson $\mathbf{P}$, Hideshima T, Anderson K. Thalidomide: emerging role in cancer medicine. Annu Rev Med 2002; 53: 629-657

23 Eleutherakis-Papaiakovou V, Bamias A, Dimopoulos MA. Thalidomide in cancer medicine. Ann Oncol 2004; 15: 1151-1160

24 Bruno B, Rotta M, Giaccone L, Massaia M, Bertola A, Palumbo A, Boccadoro M. New drugs for treatment of multiple myeloma. Lancet Oncol 2004; 5: 430-442

25 Fidler IJ, Ellis LM. Neoplastic angiogenesis--not all blood vessels are created equal. N Engl J Med 2004; 351: 215-216

S- Editor Wang GP L- Editor Wang XL E- Editor Liu WF 